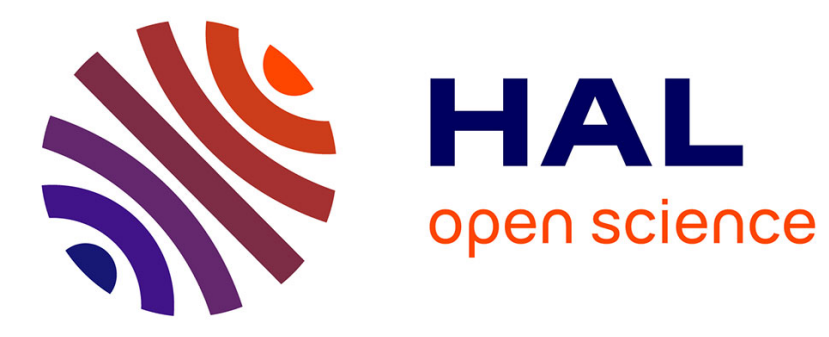

\title{
Verified Cryptographic Implementations for TLS
}

Karthikeyan Bhargavan, Ricardo Corin, Cédric Fournet, Eugen Zalinescu

\section{To cite this version:}

Karthikeyan Bhargavan, Ricardo Corin, Cédric Fournet, Eugen Zalinescu. Verified Cryptographic Implementations for TLS. ACM Transactions on Information and System Security, 2012, 15 (1), pp.3:1-3:32. 10.1145/2133375.2133378 . hal-00863381

\section{HAL Id: hal-00863381 \\ https://hal.inria.fr/hal-00863381}

Submitted on 4 Apr 2016

HAL is a multi-disciplinary open access archive for the deposit and dissemination of scientific research documents, whether they are published or not. The documents may come from teaching and research institutions in France or abroad, or from public or private research centers.
L'archive ouverte pluridisciplinaire HAL, est destinée au dépôt et à la diffusion de documents scientifiques de niveau recherche, publiés ou non, émanant des établissements d'enseignement et de recherche français ou étrangers, des laboratoires publics ou privés. 


\title{
Verified Cryptographic Implementations for TLS ${ }^{1}$
}

\author{
Karthikeyan Bhargavan \\ Microsoft Research, Cambridge \\ and \\ Ricardo Corin \\ MSR-INRIA Joint Centre, Orsay \\ and \\ Cédric Fournet \\ Microsoft Research, Cambridge \\ and \\ Eugen Zălinescu \\ MSR-INRIA Joint Centre, Orsay
}

\begin{abstract}
We intend to narrow the gap between concrete implementations of cryptographic protocols and their verified models. We develop and verify a small functional implementation of the Transport Layer Security protocol (TLS 1.0). We make use of the same executable code for interoperability testing against mainstream implementations, for automated symbolic cryptographic verification, and for automated computational cryptographic verification. We rely on a combination of recent tools, and we also develop a new tool for extracting computational models from executable code. We obtain strong security guarantees for TLS as used in typical deployments.

Categories and Subject Descriptors: C.2.0 [Computer-Communication Networks]: Security and Protection; C.2.2 [Computer-Communication Networks]: Network Protocols; D.2.4 [Software Engineering]: Software/Program Verification; F.3.1 [Logics and Meanings of Programs]: Specifying and Verifying and Reasoning about Programs

General Terms: Security, Verification
\end{abstract}

\section{VERIFYING PROTOCOLS AND THEIR IMPLEMENTATIONS}

There has been much recent progress in formal methods and tools for cryptography, enabling the automated verification of complex security protocols. In practice, however, these methods and tools remain difficult to apply. Often, verification occurs independently of the development process, rather than during design, prototyping, and testing. Also, as a protocol or its implementations evolve, it is difficult to carry over security guarantees from past formal verification. Moreover, the verification of a system that uses a given protocol involves more than the cryptographic verification of an abstract model; it may rely as well on more standard analyses of code (e.g. to ensure memory safety) and system configuration (e.g. to enforce policy). For these reasons, we are interested in the integration of modern cryptographic protocol verifiers into the arsenal of software testing and verification tools.

Symbolic vs Computational Cryptography. Two complementary approaches have been successfully applied to protocol verification.

\footnotetext{
${ }^{1}$ An earlier version of this paper appears in the proceedings of the 15th ACM Conference on Computer and Communications Security (CCS 2008).
} 
- Symbolic models treat cryptographic primitives as black boxes and focus on the logical properties of a protocol, as pioneered by Needham and Schroeder [1978] and formalized by Dolev and Yao [1983]. They have led to efficient automated tools (e.g. Blanchet [2001]; Armando et al. [2005]) that have been widely applied to the verification of large protocols.

- Computational models tackle cryptographic assumptions more concretely; they treat primitives as probabilistic algorithms over concrete bitstrings, and reason about the advantage of an adversary with bounded computational capabilities. Computational verification methods do not scale up as well as symbolic ones, sometimes leading to long, delicate, hand-crafted proofs. Automatable proof methods are more recent (e.g. Laud [2005]; Backes and Laud [2006]) and practical verification tools are just emerging (Blanchet [2006]; Blanchet and Pointcheval [2006]; Tsahhirov and Laud [2007]).

Implementations vs Abstract Models. Protocol specifications include many details; most (but not all) of them are of no importance for security. In the process of distilling a formal cryptographic model, most of these details are discarded. When is a protocol model oversimplified? In contrast with formal guarantees proved within the model, the relevance of the model relies on the experience of the formalist. The problem is compounded when considering protocol implementations. Thus, as far as possible, we propose to verify detailed protocol implementations and deployments, rather than handwritten abstract models. Using automated tools based on sound proof techniques, the details can either be safely erased, or dealt with by brute-force analysis.

From Implementations to Cryptographic Models. More recent works advocate the automatic extraction and verification of symbolic cryptographic models from executable code [Goubault-Larrecq and Parrennes 2005; Bhargavan et al. 2006].

Bhargavan et al. [2006] verify protocol implementations written in F\# [Syme 2005], a dialect of ML, by compilation to symbolic models in ProVerif [Blanchet 2001]. Their approach is to verify as much protocol code as possible, while providing hand-written models for the rest, such as the core libraries that provide cryptographic primitives, using bitstrings for concrete execution and symbolic terms for verification.

In this work, we rely on their tools for symbolic verification, and also experiment with direct computational cryptographic verifications of protocol implementations, by compilation to CryptoVerif, a recent tool for computational cryptography [Blanchet 2006].

Our Approach. Figure 1 illustrates our general approach to developing, testing, and verifying a reference implementation of a protocol plus a typical application. In contrast with the use of specialized modelling languages for cryptographic protocols, our use of a standard development platform enables early testing, for instance to disambiguate the specification, to confirm functional correctness, and to experiment with potential attacks.

Verification consists of selecting a part of the implementation, writing additional "verification harness" code that specifies the attacker model, the cryptographic assumptions, and the target security properties, and then compiling their combination to some automated prover. Since the verification tool chain is automated, one can easily re-verify the code base as it evolves, much like regression testing.

In our experience, symbolic and computational verifications are complementary. Computational verification is more precise but also more difficult to achieve; we obtain results 


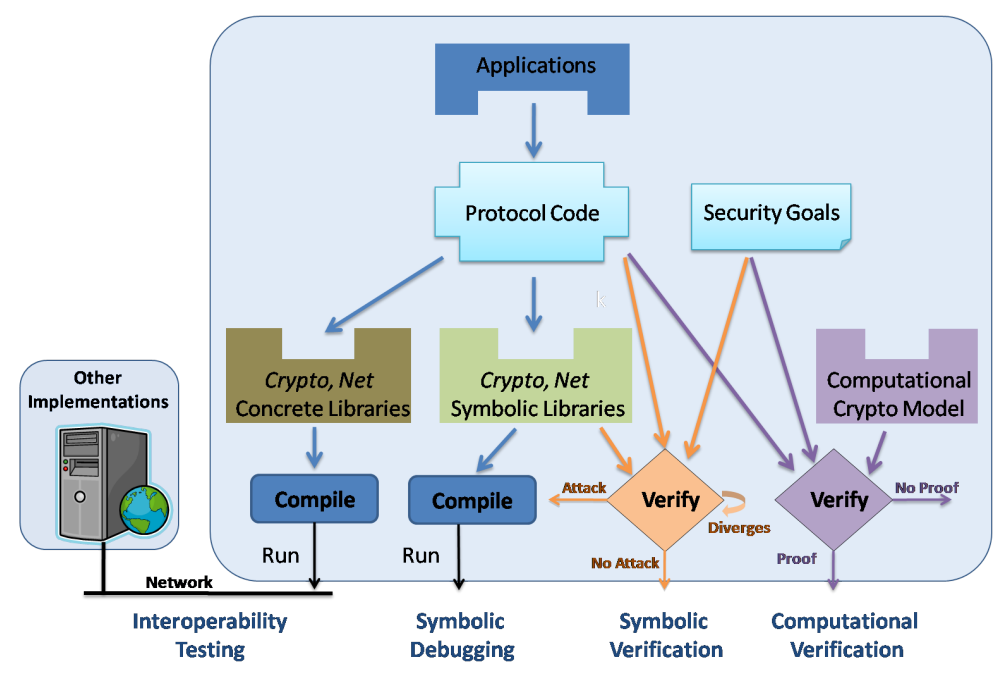

Fig. 1. Verification Architecture and Tools

only for the cryptographic core of the protocol implementation. Symbolic verification typically applies to the whole protocol, sometimes even including the application, but does not detect low-level cryptographic errors. Overall, we believe that the overhead of formal verification is getting affordable, in comparison with design, development, and testing. The next generation of tools could enable their integration in the development process.

Implementing \& Verifying TLS. As an extended case study, this paper considers implementations of TLS 1.0, one of the most widely deployed communications protocols. Due to its popularity, many systems embed an implementation of TLS and rely on its security for communications. As well as being of practical importance, TLS is a well-understood protocol, with a carefully written, self-contained specification, a series of successive versions, and a large body of related verification work, providing a detailed history of security vulnerabilities and improvements. Also, TLS is not an academic protocol, optimized (or designed) for verification purposes. This sometimes complicates its security analysis, but also provides a good benchmark for assessing verification techniques.

\section{Contributions.}

(1) We program a small functional implementation of TLS in F\#. Using simple client and server code, we confirm that it interoperates with mainstream implementations.

(2) Relying on a combination of model-extraction and verification tools, we obtain a range of positive security results, covering both symbolic and computational cryptographic aspects of the protocol. We thus provide security guarantees for code as it is used in typical deployments of TLS.

(3) To support computational verification, we develop a new tool for extracting cryptographic models from F\# code. To our knowledge, this enables the first automated verification of executable code under standard cryptographic assumptions.

(4) We review known weaknesses for earlier variants of TLS, and confirm that they are exposed as we attempt to verify the corresponding weakened variants of our code. 
Contents. Section 2 recalls the main security features of TLS. Section 3 discusses reference protocol implementations in ML. Section 4 outlines our implementation for TLS, describes its main modules, and reports on interoperability. Section 5 presents our results using symbolic models; it also discusses formal security issues and related work. Section 6 describes a new tool for extracting computational models and relating computational security assumptions to concrete cryptography APIs. Section 7 presents our results using computational models of TLS; it also discusses cryptographic issues and related work. Section 8 concludes and suggests future work.

The code for our TLS implementation and supporting libraries for its verification is available online at http://msr-inria.inria.fr/projects/sec/fs $2 \mathrm{cv}$.

\section{TRANSPORT LAYER SECURITY PROTOCOLS (REVIEW)}

The Secure Session Layer (SSL) protocol was promoted by Netscape as a means of providing privacy over the Internet, by securing HTTP connections between web browsers and servers. Its first public version, SSL 2.0 [Hickman 1995], was released in 1994. Its successor, SSL 3.0 [Frier et al. 1996], includes major changes and addresses serious security flaws. It then evolved into an Internet standard, named Transport Layer Security (TLS 1.0) [Dierks and Allen 1999]. Two more recent versions of the standard, TLS 1.1 and TLS 1.2 [Dierks and Rescorla 2006; Dierks and Rescorla 2008], include further improvements and clarifications, notably changes to thwart new cryptographic attacks. Since the three versions of TLS are relatively similar, we refer to them generically as the TLS protocol(s).

To facilitate interoperability tests, our code targets mostly TLS 1.0, the latest widelydeployed version of the protocol. Next, we recall its main security features, at the level of detail needed for the rest of the paper. We follow the notations and terminology of the standard [Dierks and Allen 1999]; we refer to it for a more general presentation.

TLS provides secure communications between a client and a server, with certificatebased authentication of the server and, optionally, of the client. The protocol distinguishes between sessions and connections; from an established session, each party can derive one or more connections, and use them to send and receive sequences of messages. The protocol has two layers. The lower layer consists of the Record protocol, for exchanging messages using current connection parameters. The upper layer includes the Handshake protocol for establishing sessions, the Alert protocol for communicating error messages, as well as application protocols.

\subsection{Record Protocol}

The Record protocol receives uninterpreted data from the upper layer. This data is first (possibly) compressed and split, then formatted into a series of records, and passed to a lower, reliable but unprotected transport protocol, such as TCP.

Both parties independently maintain state for the read and write directions of the connection. Each record is protected depending on the security parameters negotiated by the Handshake protocol, which mostly include a ciphersuite, and on the current connection state (e.g. keys and IVs). A ciphersuite specifies a key exchange mode (either DiffieHellman- or RSA-based), an encryption algorithm, and a hash algorithm. The encryption and hash algorithms are relevant only to the Record protocol, while the key exchange mode is relevant only to the Handshake protocol.

Initially, the ciphersuite is set to null, indicating no security transformations. Thus, the messages of the Handshake protocol are not protected by the Record protocol, until shared 
security parameters can be established. After the handshake, each fragment is protected using the mac-then-encrypt technique, and prefixing the result with a record header. The record header has three fields: the content type of the sub-protocol the fragment belongs to, the version of the protocol used for processing this record, and the fragment length. The mac is computed by applying HMAC [Bellare et al. 1996] (with the hash algorithm and hash secret given by the security parameters and current state) to the concatenation of the fragment, the record header, and the record sequence number. The fragment and the resulting mac are then fed to the encryption algorithm, in cipher block chaining (CBC) mode, after padding to a length that is a multiple of the block size.

\subsection{Handshake Protocol}

The Handshake protocol authenticates the server, optionally authenticates the client, establishes a shared master secret, derives cryptographic materials for their connections, and confirms that both parties agree on their exchanged parameters. In this paper, we consider only RSA-based modes. We begin with the message flow for a handshake with an anonymous client:

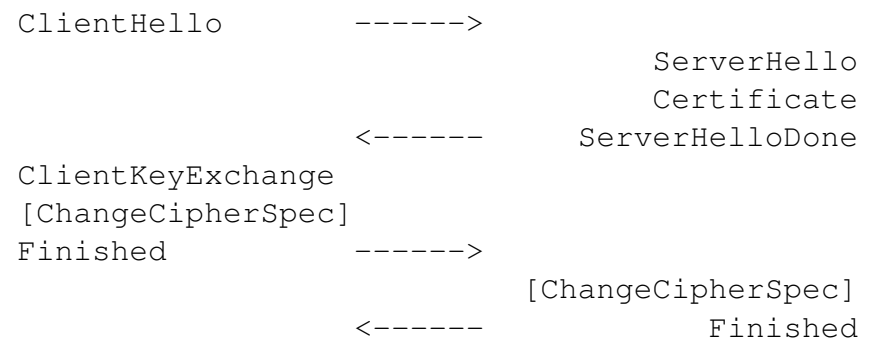

For our discussion, it is convenient to decompose the protocol into four phases, explained in more detail below.

(1) The client and server exchange connection parameters by means of the hello messages.

(2) They establish an intermediate shared pre_master_secret (pms); when using RSA, the client chooses pms, so the phase consists of a single ClientKeyExchange message.

(3) They each compute a shared master_secret (ms); this enables the record layer to derive fresh cryptographic materials for each direction of the Record protocol.

(4) They exchange ChangeCipherSpec messages, immediately followed by Finished messages, to confirm that they share matching keys, check server authentication, and ensure integrity of the handshake messages.

The Hello messages include fresh nonces, a session identifier picked by the server, and session parameters; their logical content is

ClientHello(ver_max, cr, rsid, cipher_suites, comp_methods)

ServerHello(version, sr, sid, cipher_suite, comp_method)

The Certificate message carries the server's X.509 certificate; the ServerHelloDone message has no payload.

TLS enables the negotiation of some connection parameters, that is, a protocol version, a ciphersuite, and a compression method. These parameters are passed unprotected in the Hello messages: the client expresses its preference as a range of parameters, then the server 
sets the session parameters within that range. The negotiation is authenticated later by the Finished messages, whose protection itself depends on these parameters. This circularity is a source of concerns for TLS, discussed in Section 5.

The client announces its highest supported version in the ver_max field of ClientHello and includes its lowest supported version (ver_min) in the version field of the record header that encloses ClientHello (provisionally using this version record format, for backward compatibility). The server announces its choice in the version field of ServerHello, and also includes it in the enclosing record header.

The ClientKeyExchange message includes the RSA encryption of a fresh random pms, using the public key of the received certificate. In order to confirm the highest version supported by the client, the protocol version ver_max influences the padding before RSA encryption, and is also embedded as the first two bytes of pms:

$$
\text { pms }=\text { ver_max } \mid \text { random }
$$

where $\mid$ is bitstring concatenation and random consists of 46 random bytes.

From the pms and exchanged random values, both parties compute the master secret using an ad hoc pseudo-random function (PRF). This function takes as input a secret, an identifying label, and a seed, and generates a stream of bytes, using HMAC (with two hash algorithms, MD5 and SHA1) as base primitive. For generating ms, the secret is pms and the seed is the concatenation of a fixed bitstring and the two nonces exchanged in the hello phase:

$$
\mathrm{ms}=\operatorname{PRF}(\mathrm{pms}, \text { “master secret”, } \mathrm{cr} \mid \mathrm{sr})
$$

The materials for the Record protocol are generated similarly:

$$
\text { key_block = PRF(ms, “key expansion”, sr | cr ) }
$$

is truncated and split into six secrets for the initial read and write connections: two encryption keys, two mac secrets, and two IVs.

The two ChangeCipherSpec messages appear in brackets because they are not considered part of the Handshake protocol. They signal the use of the newly-negotiated algorithms and keys, so the Finished messages are the first to be maced-then-encrypted by the Record protocol, thereby providing key confirmation. These Finished messages contain a (hashed) transcript of the handshake to this point; their logical contents is

$$
\text { verify_data }=\text { PRF(ms, finished_label, MD5(hsm) } \mid \text { SHA1(hsm)) }
$$

where finished_label is a constant string (either "client finished" or "server finished" depending on the sender of the Finished message) and hsm is the concatenation of the sequence of handshake messages (including Handshake subprotocol headers, but not the outer TLS record headers). The resulting authentication guarantees are detailed in Section 5. After a successful handshake, the parties can start exchanging application data in both directions.

\subsection{Abbreviated Handshake Protocol (Resumption)}

Instead of performing a full handshake, TLS offers the possibility of resuming a previously established session, and even of duplicating an existing session, in order to derive further connections.

ACM Journal Name, Vol. V, No. N, March 2009. 
Assume that the parties have already performed a successful handshake, thus establishing a session. The client can propose an abbreviated handshake by sending an Hello message that includes a fresh nonce and the old session identifier. If the server accepts this session identifier, both parties skip phases (2) and (3), immediately derive fresh cryptographic materials, and exchange Finished messages. Thus, the message flow for the abbreviated handshake is:

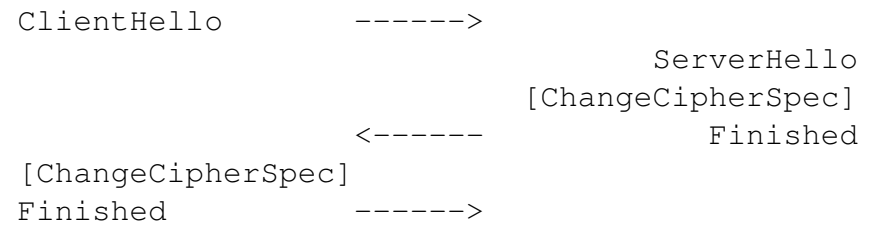

Otherwise, the server generates a fresh session identifier and the handshake continues as in the general case. The standard does not mandate whether the original connection should be used to protect the resumption; experimentally, we observe that common server implementations of TLS accept resumption ClientHello messages either protected with the current security parameters or sent in the clear.

\section{PROTOCOL IMPLEMENTATIONS IN ML}

We write our protocol implementations in F\# [Syme 2005], a dialect of ML, and execute them on the .NET runtime [Microsoft 2002]. The structure and style of our programs reflect our goal to use the same code, as far as possible, for four different tasks, as depicted in Figure 1: concrete execution, for interoperability testing; symbolic execution, for debugging; symbolic verification; and computational verification.

For symbolic debugging and verification, every .NET function or operating system call that appears in protocol or application code must be given a symbolic model. Similarly, for computational verification, such function calls must be given a computational interpretation. We identify a set of commonly-used functions and collect them in a set of core library modules written in $\mathrm{F \# .} \mathrm{We} \mathrm{require} \mathrm{that} \mathrm{protocol} \mathrm{implementations} \mathrm{use} \mathrm{only} \mathrm{these}$ library functions to interact with the .NET runtime and operating system. For each library function, we define both symbolic and computation models that are used to extract full symbolic and computational models from protocol implementations.

Our model extraction tools only support a subset of rich language features of F\#. In the remainder of this section, we describe the core library modules and language features used by our TLS implementations and supported by our tools.

Libraries for Networking and Cryptography. The module Net defines functions to set up and use TCP connections.

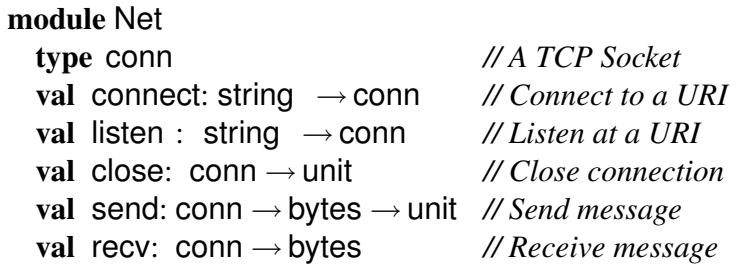


For example, by calling connect with a URI $u$, a client application can create a TCP socket to a server listening at $u$. It can then call the functions send and recv to exchange messages on this connection.

The module Crypto defines standard cryptographic primitives.

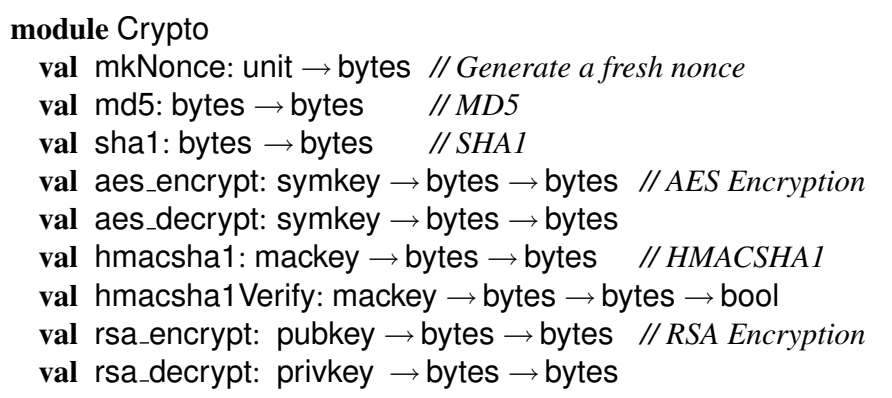

For example, our implementation of the Record protocol calls hmacsha1 and aes_encrypt to mac-then-encrypt messages, while the Handshake implementation creates a fresh pms using mkNonce and encrypts it using rsa_encrypt.

The module Prins (for principals) defines functions to create and retrieve X.509 certificates.

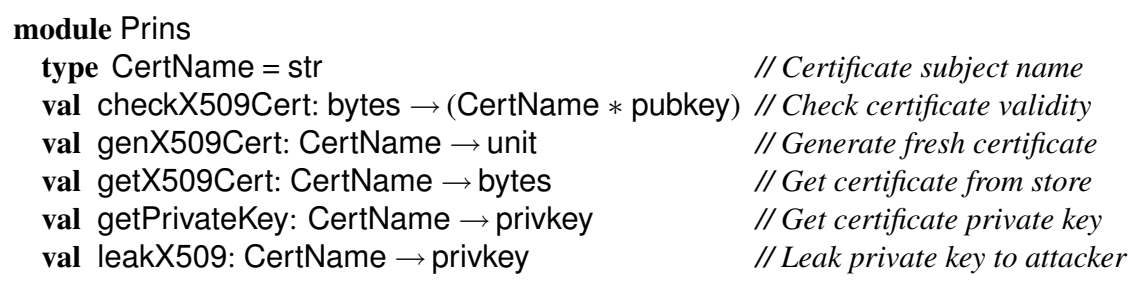

Each module has a public and a private interface. Public interfaces are those offered to applications and are also used for symbolic verification. Private ones are only used within our reference implementation. For example, the private interface of the Prins module is as above, whereas the public interface does not have the getPrivateKey function. (Hence, the attacker may only obtain private keys through leakX509.) Unless mentioned explicitly, we assume that a module's public and private interfaces coincide.

Our concrete implementation relies on various classes in the .NET Framework; for instance, the Crypto module implements hmacsha1 by calling the ComputeHash method in System.Security.Cryptography.HMACSHA1 and Net implements connect by using System .Net.Sockets.TcpClient.

Library Models for Debugging and Verification. Following an approach proposed by Bhargavan et al. [2006] (see Figure 1), we also develop a symbolic implementation of these libraries, for use in symbolic verification and debugging. In this version, the Crypto module models hashing and encryption as algebraic datatype constructors for an abstract type; for instance, hmacsha1(key,text) simply returns a term HMACSHA1(key,text) that represents the keyed hash; Net models connections as communications on local channels between processes; and Prins models the X.509 store as a local private database.

For computational verification, we develop a third version of these library functions, encoding our computational cryptographic assumptions in the source language of CryptoVerif, as described in Section 6.2. 

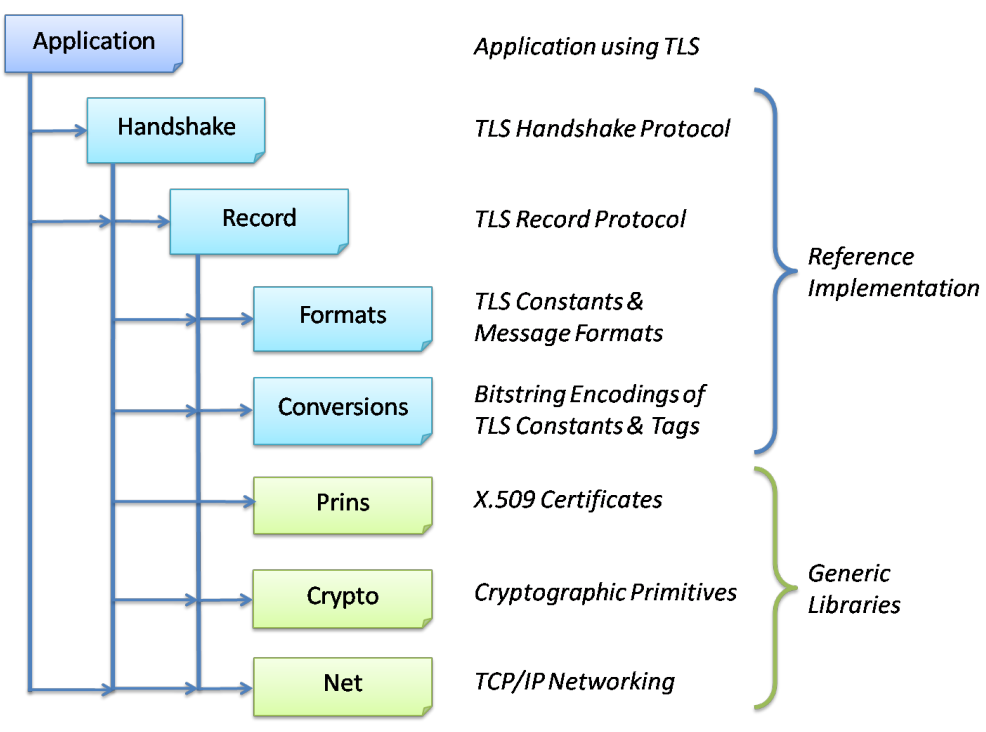

Fig. 2. Structure and Module Dependencies for our Implementation

All three versions of the library modules implement the same interfaces. By compiling a protocol implementation and application against the concrete libraries, we obtain an executable that can be deployed on the network and tested against remote clients and servers. By compiling against the symbolic libraries, we obtain an executable that can be used for generating symbolic traces for local debugging. For symbolic (and computational) verification, we assume that the concrete implementation of these libraries follows their models; as such, these libraries represent the trusted computing base for our verification results.

Supported F\# Language Features. The subset of F\# supported by our verification tools is rich enough to write modular code that accounts for detailed message formats, cryptographic operations, and security events. Our code uses typical functional language features such as nested function applications, tuples, records, algebraic datatypes, pattern matching, exceptions, and modules. However, it avoids other features such as mutable references, higher-order functions, and classes, because they are not presently supported by our verification tools. Moreover, as mentioned above, every .NET function or operating system call that we use must be included in the libraries described above. For example, in order to use file I/O operations, we would need to extend our libraries.

Our code uses recursive functions sparingly, because they usually lead to non-terminating runs of ProVerif, and are difficult to verify in CryptoVerif. For example, we have a recursive list membership function for lists of publicly known elements, but no list concatenation over private data. Moreover, we tend to separate purely functional code from code that has side effects, such as events or networks operations. Pure functions are efficiently translated to our target verification tools (for instance into ProVerif reductions), whereas functions with side effects are translated to processes, which are more complex to verify. 
messages, they log the following security events:

Send(entity, payload, conn)

$\operatorname{Recv}($ entity, payload, conn)

where entity is a label with value either Client or Server. The first event logs that entity sends message payload over connection conn. The second event logs that entity accepts message payload as valid over connection conn (after cryptographic record processing, before passing it to the application). These events have no effect at runtime; they are used only to specify our security goals for verification.

To illustrate our coding style, we detail the code for the recv function, which takes one argument, a connection identifier connid, and returns a record payload msg.

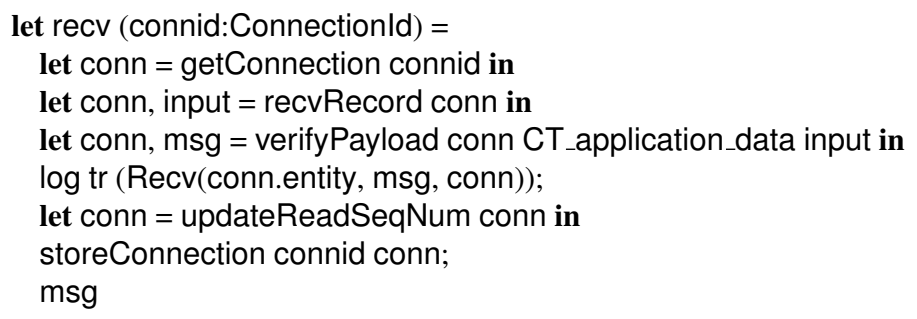

The function is written as a sequence of function calls. It first calls getConnection to retrieve the connection record conn; it then calls recvRecord, which blocks until the next message input is received on the connection; it calls verifyPayload (detailed below) to decrypt the payload msg and verify the mac; it calls log to log a Recv event as described above; it calls updateReadSeqNum to increment the sequence number of the read connection state; and it finally calls storeConnection to update the connection database with the new connection parameters before returning msg.

The cryptographic checks are all performed in the verifyPayload function:

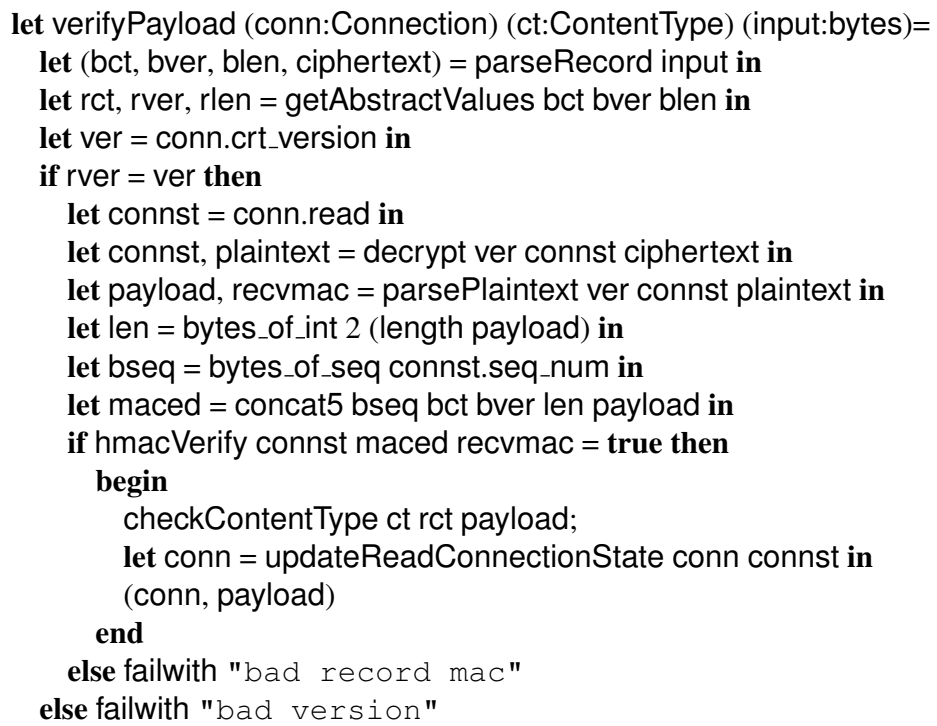

The function takes three arguments: a connection record conn, an expected content type, and a message input received over conn, and returns an updated connection conn and the 
received message payload. Most of the function prepares materials for calling the two cryptographic functions decrypt and hmacVerify. The call to decrypt decrypts ciphertext using the algorithm, key, and initialization vector stored in the connection read state connst, and yields plaintext and a new connection state with an updated initialization vector. The decrypted plaintext consists of a payload and a mac recvmac. The call to hmacVerify verifies the mac, using the algorithm and mac secret in connst, thereby authenticating the sequence number, content type, protocol version, ciphertext length, and payload. Finally checkContentType, besides checking the content type of the received record, handles the case when an alert is received.

The function fails with an exception if the mac is incorrect, if the version, content type, or sequence number do not match the expected values, if the message is an alert, or if any parsing function fails. In all other cases, it returns an updated connection and payload.

\subsection{Handshake Module}

The Handshake module exports four functions that enable client and server applications to set up new sessions, resume old sessions, and close connections:

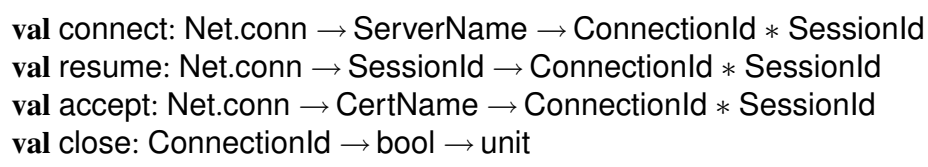

The second parameter for close is a Boolean that indicates whether to wait for the TCP connection to be terminated or not. The usage of the other functions is detailed below.

The module maintains a private database of active sessions, indexed by Sessionlds. The type Session characterizes a TLS session:

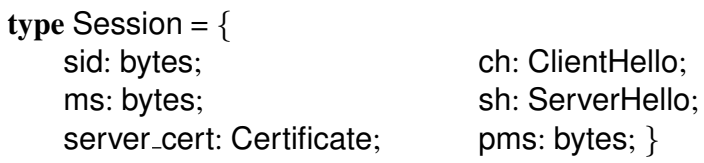

It is a record of a session identifier sid, the master secret ms, the server certificate, the client and server Hello messages, and the pre master secret pms. All these fields are exchanged during the full handshake that establish the session. The fields in the left column suffice to run the protocol; the other fields are included only for the security analysis.

A server calls accept to listen on a TCP connection for a TLS connection request; when a client calls connect over the same TCP connection, the client and server engage in a full handshake to establish a new session and a new connection in each direction. Upon completion of a full handshake, both accept and connect construct their own Session and Connection records and populate them with all the values authenticated by the handshake, including, for instance, the session identifier, ciphersuite, security parameters, and computed keys. To indicate completion of the protocol and agreement on these values, the two functions $\log$ the following events

SendFinished(entity, session, conn)

AcceptFinished(entity, session, conn)

Each event contains the entity that logs it, and full Session and Connection records. These are the records that are stored in the private databases, and of which indexes are returned by the connect and accept functions.

ACM Journal Name, Vol. V, No. N, March 2009. 
A client can call resume for stored sessions to start an abbreviated handshake. A server executing accept can also perform an abbreviated handshake if the clients asks for resumption and the requested session is stored in the server's database of active sessions. Upon completion of the Resumption protocol, both accept and resume construct their own new Connection records and populate them with the new negotiated values, that is the version and the freshly computed keys. To indicate completion of an abbreviated handshake and agreement on these values, the two functions log the following events

SendFinishedRes(entity, session, conn, ch, sh)

AcceptFinishedRes(entity, session, conn, ch, sh)

The parameters of these events have the same meaning as for a full handshake. In addition, the client and server Hello records are tracked. In these events, all fields in session (including for example session.ch and session.sh) refer to the initial full handshake, while the last two parameters ch and sh refer to the Hello messages in the abbreviated handshake.

\subsection{Sample Applications and Interoperability}

Using our reference implementation, we write three applications:

- a client that connects to an HTTPS URI and retrieves a web page over a TLS connection;

- a server that listens at an HTTPS URI and returns a web page over a TLS connection;

- a mutually authenticated client-server application where the client authenticates to the server using a password over a TLS connection.

Next, we outline the code for the two client applications, and we describe how we test them symbolically and concretely.

Simple HTTPS Client. Our basic HTTPS client uses functions from the Handshake and Record modules. The function clientHttps fetches a single page from a server, by calling the auxiliary functions tlsconnect and httpGet:

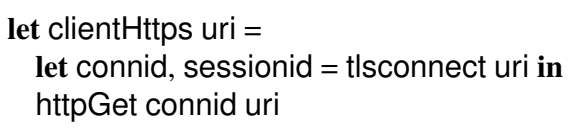

The function tIsconnect first establishes a TCP connection (conn) with the server by calling Net.connect and then calls Handshake.connect to perform a TLS handshake over this unprotected connection:

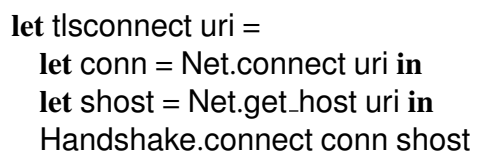

If the handshake succeeds, it returns a connection identifier (connid) and a session identifier (sessionid). The function httpGet performs an HTTP exchange over the TLS connection. It builds an HTTP/Get request, sends the request over the TLS connection, waits for a response, parse it, and return its contents:

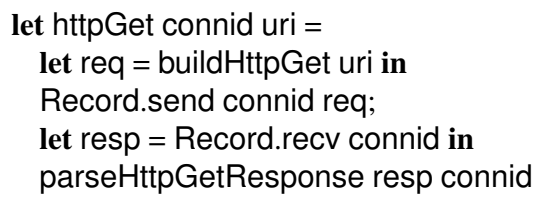


Password-based Client Authentication. To illustrate how applications with additional security properties may be built on top of our TLS implementation, we develop a client-server application where the client sends its user name and password over a TLS connection, and the server checks these values to authenticate the client. We assume that the client passwords are already shared between clients and servers, and stored in a private database on the server side. The code for the client is as follows:

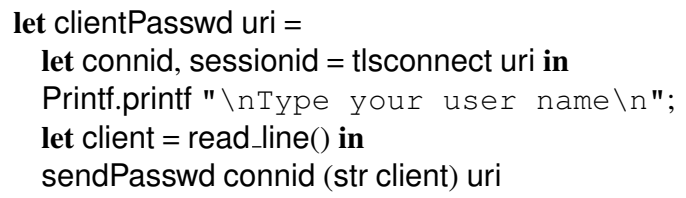

As in the simple HTTPS application, the client calls tlsconnect to establish a TLS connection. It then reads the user name from a console and calls the function sendPasswd that performs a secure message exchange with the server.

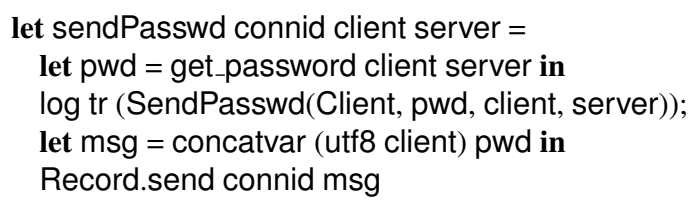

The get_password function returns a bitstring and may be implemented in several ways. For instance, it may ask the user to enter her password, or it may query a password database. (Our symbolic verification does the latter.) The client logs the password with the identities of the involved parties, and sends its user name and password over the Record protocol. (The function concatvar prepends a length field to the concatenated bitstring so that the server may unambiguously parse the record payload.)

Concrete Executions and Interoperability Testing. As a first experiment, we run our applications concretely over a network and test them against one another. For instance, our HTTPS client and server are successfully able to perform both handshake and resumption protocols, and to perform HTTP exchanges on top of the established connections.

Then to test interoperability, we run our applications concretely over a network against popular client/server applications. In our experiments, our simple HTTPS client application successfully fetches pages from web servers running Microsoft IIS 7.0 or Apache 2.2.9; and our HTTPS server application successfully serves pages to clients running Microsoft Internet Explorer 7 or Mozilla Firefox 3.0.6. Both applications successfully resume sessions when asked, for instance, to refresh a web page displayed in a previous session. Hence, at least for these applications, our reference implementation interoperates with the mainstream TLS implementations used by these browsers and web servers, including OpenSSL and the Windows CryptoAPI.

In practice, most TLS implementations expose only a selection of cryptographic suites, and sometimes slightly deviate from the standard, for instance in the handling of the versions in ClientHello and ClientKeyExchange messages. Our experiments also helped us explore these issues and disambiguate details of the standard.

In comparison to mainstream implementations, our reference implementation supports a smaller subset of the standard. We focus on TLS 1.0 in RSA mode for the key exchange, and provide partial support for SSL 3.0 and TLS 1.1. In this mode, we support all ciphersuites using AES, DES, RC4, SHA, and MD5 algorithms, thus for example 
TLS_RSA_WITH_AES_128_CBC_SHA and TLS_RSA_WITH_RC4_128_MD5. Our implementation does not support data compression, nor fragmentation; it does not send alerts, and it silently fails upon receiving a bad message. Despite these limitations, our experiments show that it is adequate for writing simple client and server applications.

Symbolic executions. We can also run our applications linked with symbolic versions of our libraries. Communication is then performed locally, between client and server threads, using abstract terms instead of bitstrings. For instance, the ClientHello message becomes

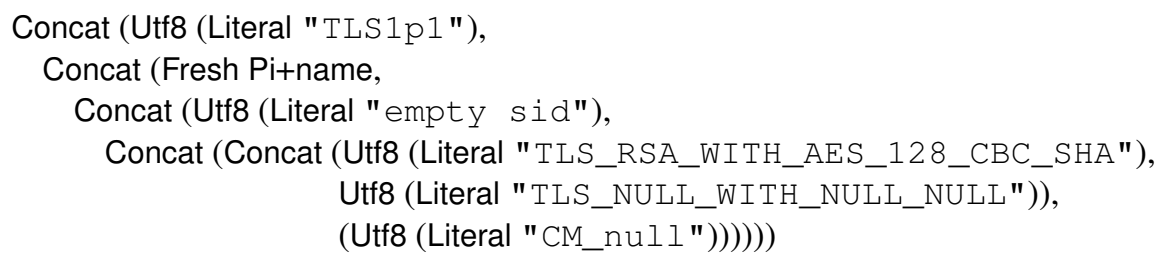

where the ML datatype constructors Concat, Utf8, Literal, Fresh, Pi+name are seen here as term constructors, and strings are seen as term constants.

Symbolic message traces are especially useful for debugging. For instance, an error in the sequence of concatenations above would make the connection fail, but would be difficult to detect from encoded bitstrings exchanged in concrete executions. Symbolic traces help find such errors by inspection by revealing the logical structure of messages.

\section{SYMBOLIC VERIFICATION}

Our symbolic verification is based on an existing tool chain consisting of a model extractor [Bhargavan et al. 2006], that compiles code written in F\# to process models in an applied pi calculus [Abadi and Fournet 2001], and the state-of-the-art verifier ProVerif [Blanchet 2001; Blanchet 2002], that analyzes such models automatically. Although our symbolic verification problem is undecidable in general, for many protocol implementations, the verifier either proves the security goals or produces a counter-example. In some cases, the verifier may not terminate; in others, it may take several gigabytes of memory.

To use this tool chain, we write symbolic implementations for selected low-level libraries, as described in Section 3; we define the attacker model in terms of the public interface exposed by these libraries and by our reference implementation; and we write our authentication and secrecy goals as correspondences between events logged by functions in the interface. Then, we can extract a symbolic model from the reference implementation and verify security queries automatically. If the tool proves a query, we obtain a security theorem about the protocol implementation, against all attackers that use its public interface. Our results rely on the correctness of the core translations and algorithms underpinning the model extractor and ProVerif [Bhargavan et al. 2006; Blanchet 2001; Blanchet 2002].

Attacker Model. The attacker capabilities are given by the public interfaces of the modules Net, Crypto, Prins, Handshake, and Record. Thus, we let attackers range over arbitrary programs that use the functions given in these interfaces. This yields a rich symbolic threat model à la Dolev and Yao [1983] with an active attacker that can

- control the network (Net) and perform cryptographic operations (Crypto);

- create any number of servers by generating certificates and, optionally, compromise any server by reading its private key-whenever this occurs, an event Leak(subj) is emitted to record the subject subj of the compromised certificate (Prins); 
- run any number of sessions between clients and servers of its choice, obtaining their connection and session identifiers; and trigger the Resumption protocol for any session identifier (Handshake);

—send and receive messages over the record layer (Record).

We let System range over programs that consist of the symbolic implementations of the Net, Crypto, and Prins, modules, of the implementations of the Handshake, Record, Formats, and Conversions modules, and of arbitrary code with access to the public interfaces of Net, Crypto, Prins, Handshake, and Record. All the security results of this section hold for any run of any such program.

\subsection{Handshake Protocol}

We first present symbolic results for the Handshake protocol. For the Full Handshake, authentication is specified as a correspondence from events triggered when a party accepts the peer's Finished message to prior events triggered when the party sends that message. The more information these events record, the stronger the property. We say that a server has been corrupted when its private key has been leaked to the attacker. We say that a client is corrupted if its pms is known to the attacker.

THEOREM 1 (FUll HANDShaKe AUthenTICATION). In any run of System, for any AcceptFinished event logged by an entity (client or server), either there is a SendFinished event logged by the opposite entity (server or client respectively) with matching connection and session parameters, or the opposite entity is corrupted.

In these statements, the server identity is just its certificate subject; it is left to the application to correlate this authenticated identity with the intended peer server, using for instance the target URL. (Experimentally, neither Internet Explorer nor Firefox sends the client Finished message when this correlation fails.)

The proof is by automated verification of the two queries below on the extracted model.

query ev: AcceptFinished(Client, sess, conn)

$\Longrightarrow$ principals: sess, subj, pms \& correctParam: sess \& connections: conn, conn' $\&$ (ev: SendFinished(Server, sess, conn') | ev: Leak(subj)).

query ev: AcceptFinished(Server, sess, conn)

$\Longrightarrow$ principals: sess, subj, pms \& correctParam: sess \& connections: conn, conn' $\&$ (ev: SendFinished(Client, sess, conn') | attacker: pms).

In the queries, lowercase parameters are variables, whereas Client and Server are symbolic constants. Queries relate facts using logical conjunction (\&), disjunction (|), and temporal implication $(\Longrightarrow): \mathrm{e} \Longrightarrow \mathrm{e}^{\prime}$ means that whenever $\mathrm{e}$ is true, $\mathrm{e}^{\prime}$ must have been true before. Of the three connectives, \& has the highest precedence and $\Longrightarrow$ the lowest. All variables in a query are universally quantified, except for the variables on the right-hand-side of $\Longrightarrow$ that do not appear in the left hand side, which are existentially quantified. Each fact has the form $\mathrm{p}: \mathrm{m}_{1}, \ldots, \mathrm{m}_{n}$, where $\mathrm{p}$ is a predicate and $\mathrm{m}_{1}, \ldots, \mathrm{m}_{n}$ are symbolic terms.

The predicates ev and attacker are predefined in ProVerif; ev: e means that the event e has been logged, and attacker: $\mathrm{m}$ means that the attacker knows message $\mathrm{m}$.

The predicates principals, correctParam, and connections are defined by us; principals: sess, subj, pms holds when the subject of the certificate sess.server_cert, and sess.pms equal respectively subj and pms; correctParam: sess holds when sess.sid equals the sid 
field of the ServerHello message sess.sh; connections: conn,conn' holds when the version field crt_version of the two connections are equal, and when the read connection state of conn equals the write connection state of conn' (and vice-versa). We detail the definition of the predicate connections in ProVerif; the other predicates are defined similarly. This predicate is defined by the Horn clause

clauses connectionsMatch(conn,conn') $=() \rightarrow$ connections: conn,conn'.

that says that connections: conn,conn' holds when the function connectionsMatch succeeds. This F\# function is defined in the Record module and checks that the connections share the same session-identifier and version fields and have opposite entities.

The two queries require that, at the end of the handshake, the server and client agree on the important fields of the session. These fields include sid, pms, ms, server_cert, and also $\mathrm{cr}$, sr, version, and cipher_suite from ch and sh in session. The derived cryptographic materials (within the read and write connection states of conn and conn') are also correlated.

As a minor technical point, there is no formal agreement on the client's lowest supported version for TLS (ver_min): the attacker may change this value, together with the message record format for ClientHello, without detection. However, this is innocuous as long as (1) the client checks that version $\geq$ ver_min and (2) the server's choice of version does not depend on ver_min. Indeed, the standard says that the server should pick version to be either ver_max or its highest supported version, whichever is lower.

Session Resumption. We obtain a similar theorem for the Resumption protocol.

THEOREM 2 (RESUMPTION AUTHENTICATION). In any run of System, for any Accept FinishedRes event logged by an entity (client or server), either there is a SendFinishedRes event logged by the opposite entity (server or client) with matching connection and session parameters, or the opposite entity is corrupted.

Moreover, within each AcceptFinishedRes and SendFinishedRes event, the new ServerHello message has the same session identifier and ciphersuite as the old session.

The proof is by automated verification of the two queries

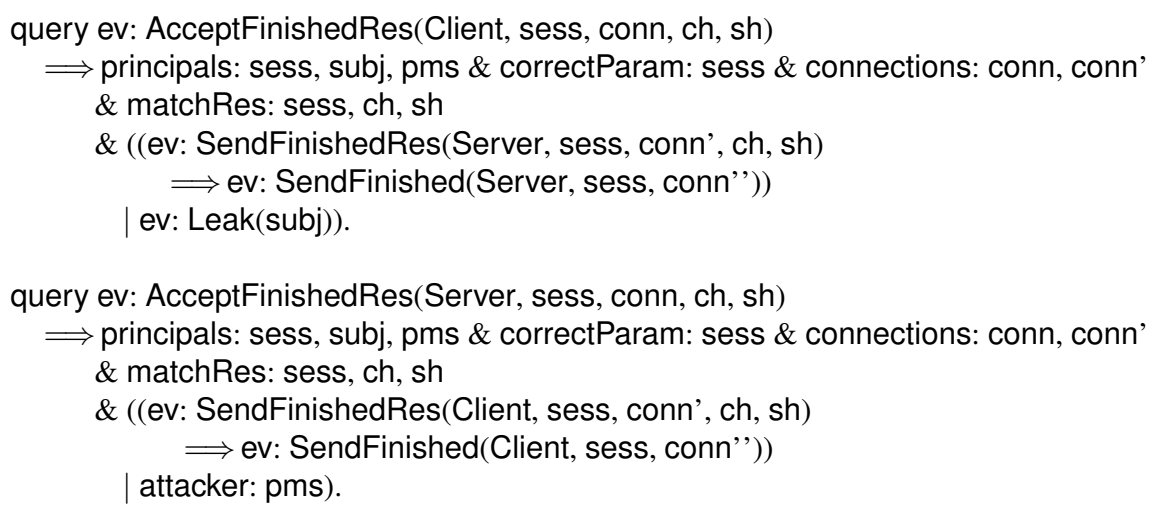

These queries use a new predicate matchRes that correlates session parameters from the original handshake (recorded in sess) with the connection parameters established by the new abbreviated handshake (recorded in the Hello messages ch and sh). In particular, matchRes requires that the old sess.sid equals the rsid requested in the new ch and the+ 
sid returned in the new sh; it also requires that the old cipher_suite (within sess.sh) equals the new cipher_suite (within sh).

Conversely, matchRes does not ask for equality between the version fields of the old and new ServerHello message, and with good reason: the above queries do not hold if matchRes also require agreement on the protocol version. As explained in Section 5.5, this reflects a potential version rollback attack on TLS resumption.

In the two queries, the second $\Longrightarrow$ is a requirement that a full handshake have been executed before resumption. For instance, when verifying the first query, whenever an AcceptFinishedRes event occurs, some SendFinishedRes event (with matching parameters) must have occurred before, and moreover, this SendFinishedRes event can only have occurred after some SendFinished event. These queries encode a weak form of client authentication: during resumption, the new connection is associated with the same client as for the initial connection; hence, an adversary cannot use resumption to hijack a session.

Handshake Secrecy. We prove secrecy for all secrets generated during full and abbreviated handshakes, including pms, ms, the two encryption keys, the two MAC secrets, and the two IVs. Our theorem asserts syntactic secrecy; it requires that the secret values are not obtained by the attacker, unless he has compromised the server or controls the client.

THEOREM 3 (HANDShaKe SECRECY). In any run of System, for any connection in any session between two entities, either the values of pms, ms, all encryption and mac keys, and initial IVs are syntactically secret, or one of the entities is corrupted.

To encode this secrecy requirement, we extend the protocol interface with a function that allows an attacker to guess the value of a secret and, if the guess matches the value stored in the connection or session database, logs an event LeakedSecret to indicate that the attacker has compromised the session and connection. Then, the theorem is established by proving the query

query ev: LeakedSecret(entity, sess, conn) $\Longrightarrow$ principals: sess, subj, pms \& (ev: Leak(subj) | attacker:pms).

stating that, if any of the secret values stored in the database is obtained by the attacker, then one of the two entities in the session must be corrupted.

\subsection{Record Protocol}

For the Record protocol, authentication is specified as a correspondence between the Recv events emitted when a receiver accepts a message and the Send events emitted when messages are sent.

THEOREM 4 (RECORD AUtHENTICATION). In any run of System, for any Recvevent logged by an entity (client or server), either there is a Send event logged by the opposite entity (server or client) with matching payload and connection parameters, or one of the two entities is corrupted.

The proof is by verification of the two queries below.

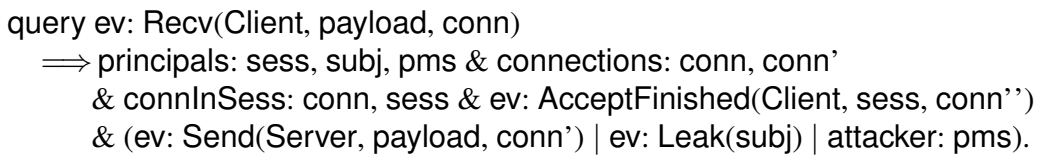

ACM Journal Name, Vol. V, No. N, March 2009. 
query ev: Recv(Server, payload, conn)

$\Longrightarrow$ principals: sess, subj, pms \& connections: conn, conn'

\& connInSess: conn, sess \& ev: AcceptFinished(Server, sess, conn'’)

$\&$ (ev: Send(Client, payload, conn') | ev: Leak(subj) | attacker: pms).

The queries say that if a Recv event is logged for a connection conn that is part of a session sess, then either the corresponding Send event was logged, or one of the entities in sess is corrupted. In contrast with handshake authentication, our record authentication theorem allows for the corruption of either entities. This is because record authentication depends upon the secrecy of session and connection secrets, specifically the authentication keys and IVs, and these are secret only if neither entity is corrupted (Theorem 3 ). Note also that the Recv event only mentions the connection conn and not the session; so both queries use the AcceptFinished event to choose some valid session sess and then call connInSess: conn, sess to check that the session identifier in sess matches the one in conn.

Record authentication implies that the client and server have the same sequence number. (read.seq_num). In TLS, sequence numbers are incremented each time a record is processed, to ensure connection integrity: if a sequence of records is received by some party on a connection, then the corresponding party has sent (at least) the same sequence of message on the same connection. Although Theorem 4 is stated in terms of a single message, it implies connection integrity in the following sense. It guarantees that, whenever a record payload is accepted, both parties agree on its sequence number. Hence, if the accepted payloads and sent payloads are arranged in order of their sequence numbers, either the accepted sequence is a prefix of the sent sequence, or the sending entity is corrupted. This argument holds for arbitrarily large sequence numbers; we support this in our symbolic model by allowing the adversary to choose sequence numbers (the sender reads the sequence number for the next record from a public channel).

Next, we show that the Record protocol preserves payload secrecy. More precisely, we prove that if a freshly generated (secret) nonce is used as a record payload over an established connection, then it remains syntactically secret as long as both client and server are uncorrupted. To specify this property, we extend the Record module with two new functions. First, we add a function send' that only sends fresh nonces as record payloads:

let send' (id:Connectionld) $=$

let record_payload $=$ mkNonce () in send id record_payload

Second, we add a function that allows the attacker to guess the value of a payload and, if the guess matches one of these fresh nonces, logs an event LeakedPayload to indicate that a secret payload has been obtained by the attacker. We add both these functions to the Record public interface and remove the function recv-otherwise, recv acts as a decryption oracle allowing the attacker to trivially obtain the secret payload. We let System ${ }^{\prime}$ be the variant of System with this modification.

THEOREM 5 (RECORD PAYLOAD SECRECY). In any run of System', for any record payload sent over a connection between two entities, either the payload is syntactically secret, or one of the entities is corrupted.

The proof is by verification of the query

query ev: LeakedPayload(entity, payload, conn) 
$\Longrightarrow$ principals: sess, subj, pms \& connInSess: conn, sess \& ev: AcceptFinished(entity, sess, conn') $\&$ (ev: Leak(subj) | attacker: pms).

This query is structurally similar to the authentication queries; it retrieves a valid session for the connection and states that either the client or the server must be corrupted.

\subsection{Password-based Client Authentication}

Section 4.3 describes an application that use shared passwords for client authentication on top of TLS connections. Clients are identified by a username and password; servers are identified as usual by the subject name of their X.509 certificates. The application is implemented in the Passwd module, and has the interface:

val generatePasswd: UserName $\rightarrow$ ServerName $\rightarrow$ unit

val clientPasswd: UserName $\rightarrow$ ServerName $\rightarrow$ unit

val serverPasswd: ServerName $\rightarrow$ unit

The function generatePasswd creates a new username-password record for use at a particular server and stores it in a protected password database. The functions clientPasswd and serverPasswd start new instances of the client and server application, respectively.

We let System ${ }^{\prime \prime}$ be System augmented with the Passwd module and arbitrary code with access to the interface of Passwd plus all the public interfaces of System.

THEOREM 6 (PASSWORD-BASED USER AUTHENTICATION). In any run of System ${ }^{\prime \prime}$, for any AcceptPasswd event logged by a server, either there is a SendPasswd event logged by a client with matching username, password, and server name, or the password is known to the attacker, or or the server is corrupted.

The proof is by verification of the query

query ev: AcceptPasswd(Server, pwd, user, subj)

$\Longrightarrow$ ev: SendPasswd(Client, pwd, user, subj)| attacker: pwd | ev: Leak(subj).

\subsection{Experimental Results}

All the queries in this section are automatically verified on the systems described above. In some cases, we had to hand-tune the protocol code extensively in order to make verification feasible-otherwise ProVerif either ran out of memory, or did not terminate for several days. To this end, during early development, we also verified parts of the protocol in isolation. For comparison, Figure 3 gives the verification times for these simpler experiments as well as for the full systems. All experiments are performed on a computer with Intel Xeon Dual Quad-core processors at $3 \mathrm{GHz}$, and 32GB RAM. We used FS2PV version 1.1 and ProVerif version 1.14pl5.

The first row of Figure 3 describes the verification of handshake authentication (Theorem 1) on just the Handshake module, with Record and Resumption protocols disabled. (These protocols are disabled by removing their functionality from the public interface.) The second row verifies handshake secrecy (Theorem 3) again for just the Handshake protocol. The third row verifies the Handshake module for resumption authentication (Theorem 2), but with the Record protocol disabled. The fourth row verifies our full reference implementation of TLS for record authentication (Theorem 4). The fifth row verifies the full implementation for all the queries in this section, establishing Theorems 1-5. The final row verifies our password-based client-server application (Theorem 6).

ACM Journal Name, Vol. V, No. N, March 2009. 


\begin{tabular}{|l|l|r|r|r|r|}
\hline Verified Parts of TLS & Security Goals & F\# Code & Queries & Time & Memory \\
\hline \hline Full Handshake & Authentication & 1418 lines & 2 & $27 \mathrm{~s}$ & $60 \mathrm{MB}$ \\
\hline Full Handshake & Secrecy & 1418 lines & 2 & $25 \mathrm{~s}$ & $80 \mathrm{MB}$ \\
\hline $\begin{array}{l}\text { Full Handshake \& } \\
\text { Resumption }\end{array}$ & Authentication & 2194 lines & 2 & $8 \mathrm{~min}$ & $460 \mathrm{MB}$ \\
\hline $\begin{array}{l}\text { Full Handshake \& } \\
\text { Resumption \& Record }\end{array}$ & $\begin{array}{l}\text { Authentication } \\
\text { (Record Only) }\end{array}$ & 3344 lines & 2 & $11 \mathrm{~min}$ & $700 \mathrm{MB}$ \\
\hline $\begin{array}{l}\text { Full Handshake \& } \\
\text { Resumption \& Record }\end{array}$ & $\begin{array}{l}\text { Authentication \& } \\
\text { Secrecy }\end{array}$ & 3344 lines & 10 & $3.5 \mathrm{~h}$ & $4.5 \mathrm{~GB}$ \\
\hline $\begin{array}{l}\text { Full TLS \& } \\
\text { Password-based Application }\end{array}$ & $\begin{array}{l}\text { User } \\
\text { Authentication }\end{array}$ & 3855 lines & 1 & $1.5 \mathrm{~h}$ & $1.2 \mathrm{~GB}$ \\
\hline
\end{tabular}

Fig. 3. Summary of experimental results for symbolic verification

\subsection{Related Work: Symbolic Attacks on TLS}

Previous formal and informal analyses of SSL and TLS have uncovered a range of vulnerabilities and attacks. For instance, SSL 2.0 does not guarantee integrity of many elements of the handshake negotiation, including the ciphersuite. Hence, if the client and server both prefer to use strong cryptography, but are also willing to use weak cryptography, then an attacker may convince them both to establish a session with weak cryptography; this is called a ciphersuite rollback attack. In our model, this attack appears as a failure of handshake authentication (Theorem 1). We experimented with an SSL 2.0-like variant of our client implementation that sends application data before receiving a valid Finished message. ProVerif rightfully fails to prove an authentication query, and instead produced a counter-example indicating the attack.

Recent versions of TLS, since SSL 3.0, guarantee full handshake integrity by authenticating all previous handshake messages in the final Finished messages. In particular, Hello messages are integrity protected thus preventing ciphersuite rollback attacks. However, if a client and server still support SSL 2.0 for backwards compatibility, then a new version rollback attack becomes possible: an attacker may convince both parties to use SSL 2.0, and then exploit any flaws (including ciphersuite rollback) of the earlier version. Version rollback is a general problem faced by protocols that have multiple versions deployed at the same time. SSL 3.0 and TLS include two mechanisms to address this problem, both in the ClientKeyExchange message, so that a server can authenticate ver_max, the highest protocol version supported by the client. In particular, TLS clients embed ver_max within pms, but SSL 2.0 clients do not. Hence, a server can identify clients that support versions higher than SSL 2.0 and protect itself from version rollback. Indeed, Theorem 1 shows that our implementation of the Handshake protocol authenticates both the lowest and highest version supported by the client.

The version rollback protection mechanisms of TLS does not suffice for the Resumption protocol, since the abbreviated handshake does not contain a ClientKeyExchange message. Hence, every implementation of TLS, including ours, is vulnerable to version rollback during resumption. In particular, resumption authentication (Theorem 2) guarantees only that the new connection parameters excluding ServerHello.version are correlated with the old session parameters, not that the new version is higher than the old one. Experimentally, we found that deployed server implementations of TLS are vulnerable to version rollback 
attacks from TLS 1.0 to SSL 3.0 during resumption. However, we could not reproduce the potentially more dangerous rollback from TLS 1.0 to SSL 2.0, partly because the length of the session identifier differs between these two versions.

In addition to protocol-level attacks, our method also finds common implementationlevel attacks. A common error in many TLS implementations and applications is the incorrect validation of received server certificates [Ruby 2006; Cisco 2007]. In an early version of our implementations, we found a certificate validation error (as a failure of Theorem 1). In other early versions, we found errors such as allowing the null ciphersuite to be selected, or not checking that the received sequence number is correct. In each case, our verification tools generated counter-examples to our secrecy and authentication queries.

More recent vulnerabilities in OpenSSL exposed over four hundred applications that relied on OpenSSL for its cryptographic and TLS/SSL functionality [OpenSSL 2009]. In essence, these applications and other functions withing OpenSSL, incorrectly handled the return values of the main signature verification functions, treating erroneous signatures as valid. In particular, the vulnerability allowed certificate verification in the OpenSSL TLS implementation to be bypassed in some conditions. Since our implementation does not use return values to signal errors and instead raises exceptions, such vulnerabilities do not directly appear in our code. However, our verification tools would find those errors as well.

On the other hand, it is worth pointing out that several well-known attacks on TLS are outside the scope of our symbolic model. These include cryptanalyses on the underlying cryptographic functions, traffic analyses, and padding attacks.

\subsection{Related Work: Previous Symbolic Analyses}

In a long line of works, starting from Dietrich [1997], researchers have used various techniques to verify models, and more recently, implementations, of different versions of SSL and TLS. We describe only those most closely related to our work.

Wagner and Schneier [1996] carry out an informal analysis of SSL 3.0. They point out an ambiguity in the specification which allows application data to be exchanged before the confirmation of security parameters with the Finished messages. Thus in this case ciphersuites rollback attacks could be successful. This ambiguity was corrected in the TLS 1.0 protocol. They also fear possible anomalies in the Resumption protocol related to version rollback issue we have mentioned.

Mitchell et al. [1998] study a model of SSL 3.0 using the Murphi tool. They use modelchecking to perform a finite-state exploration of a sequence of protocols of increasing complexity, including a version of SSL 3.0 with both full and abbreviated handshakes, but limited to finite configurations consisting of, for example, two clients and a server.

Paulson [1999] develops formal, machine-checked proofs for a model of TLS 1.0 in Isabelle, with authentication and secrecy theorems that, like ours, apply to more general configurations of clients and servers. His model includes both full handshake and resumption but does not address version rollback issues within resumption.

He et al. [2005] apply logic-based proof techniques to the IEEE 802.11i protocol, and include a simple model of TLS as a subprotocol. Using PCL, they prove agreement on all exchanged messages and secrecy of the pre-master secret. Díaz et al. [2004] show the correct message flow of the Handshake protocol in the absence of the intruder using the UPPAAL model-checker. Ogata and Futatsugi [2005] show secrecy of the pre-master secret and liveness properties for the Handshake protocol using the OTS/CafeOBJ tool. Kamil and Lowe [2008] report on an analysis of a detailed strand spaces model of the 
Handshake and Record protocols. They prove authentication and secrecy theorems similar to ours, and also show that the Record protocol provides two authenticated streams and satisfies session independence.

Jürjens [2006] verifies a Java implementation of the Handshake protocol for secrecy and authentication properties. His analysis works on the control-flow graph and does not account for multiple versions or low-level message formats. Chaki and Datta [2008] apply software model checking on OpenSSL code to verify secrecy and authentication for configurations of up to three servers and clients.

\section{A COMPUTATIONAL VERIFIER FOR PROTOCOL IMPLEMENTATIONS}

This section describes our computational verification approach and tools; Section 7 applies them to TLS. Compared with symbolic models, computational models adopt a less optimistic approach to cryptography: rather than giving the adversary essentially the same capabilities as ordinary protocol participants, they specify both minimal positive assumptions (guaranteeing, for instance, that the correct decryption of an encrypted message yields the original plaintext) and minimal negative assumptions (bounding, for instance, the probability that a polynomial adversary may break a particular usage of encryption).

\subsection{CryptoVerif (Review)}

The CryptoVerif verifier can prove the security of a given protocol under a set of security assumptions for its cryptographic primitives, within a probabilistic polynomial time (PPT) model of computation. We briefly present the tool; we refer to [Blanchet 2006] and [Blanchet and Pointcheval 2006] for an explanation of CryptoVerif syntax and semantics.

CryptoVerif takes as input a script, written in a variant of the pi calculus with an explicit polynomial bound for every replicated process. Thus, processes represent PPT Turing machines that exchange finite bitstrings through an adversary, modeled as an (unknown) PPT machine. In the script, cryptographic assumptions are introduced through type and function declarations, equations, inequations, and game-based equivalences. The equations and inequations are typically used to describe minimal positive assumptions (the functional correctness of the primitive), whilst the game-based equivalences are used to state minimal negative assumptions. Section 7 gives some examples.

Proofs, Games, and Indistinguishability. The input script can be seen as an initial game, modelling the protocol, to which CryptoVerif applies transformations, until a final game that satisfies target security conditions is reached-this proof technique is known as gamehopping [Blanchet and Pointcheval 2006; Corin and den Hartog 2006].

Each transformation between two consecutive games preserves PPT indistinguishability, that is, the adversary cannot distinguish the games before and after the transformation. Example transformations include the application of an equivalence stating the security of a cryptographic primitive, and the semantics-preserving rearrangement of code, such as inlining and partial evaluation. CryptoVerif runs either automatically or interactively, in which case it receives guidance from the user for selecting transformations.

In a recent case study, CryptoVerif is used to verify a model of the Basic and Public-Key Kerberos protocol [Blanchet et al. 2008].

Process and Variable Instances. Processes in CryptoVerif can be replicated polynomially in a given security parameter, enabling multiple parallel executions. Hence, every replica 
has access to its own instance of each variable; in addition, a special find command gives read access to all other instances of each variable.

Target Security Properties. CryptoVerif can verify authentication and secrecy properties. Authentication goals are written as correspondences [Blanchet 2007], much as in our symbolic models. Computationally, correspondences assert that, if some event is executed, then other events must also have been executed, at least once, with matching parameters, at least with overwhelming probability; this last bit reflects the computational nature of the model. (CryptoVerif can deal with more general properties expressed as logical formulas; also both injective and non-injective properties can be analyzed.)

Secrecy goals are expressed as indistinguishability between two configurations. (It is often called strong secrecy in symbolic models, in contrast with the weaker notion of syntactic secrecy.) CryptoVerif has two notions of secrecy. The weaker notion (query secret 1 in CryptoVerif) states that the adversary cannot distinguish the value of any instance of a given variable from a random value; the stronger notion (query secret) states that the adversary cannot distinguish the vector of values of all the instances of a given variable from a vector of independent random values.

\subsection{A Compiler from ML Programs to CryptoVerif Scripts}

We outline the design of our new model extractor that translates protocol implementations written in F\# to CryptoVerif scripts. (See Bhargavan et al. [2009] for additional details.) The extractor takes three inputs: protocol modules written in F\#, such as the modules in our reference implementation, a computational model of the cryptographic libraries expressed as CryptoVerif assumptions, and security goals for the protocol expressed as CryptoVerif queries. It then generates a CryptoVerif script that can be verified either automatically or interactively. In the rest of this section, we outline the various steps of the translation.

Computational Models for Libraries. We first define models for all the functions in the library modules, such as Net, Crypto, and Prins. Our model of Net treats connections as public channels; hence, calls to Net.send and Net.recv send and read messages from a single public channel that is controlled by the attacker. Our model of Crypto treats bytes as concrete bitstrings, and defines cryptographic primitives as uninterpreted functions over bitstrings. For each primitive, it defines equations, inequations, and equivalences that encode specific cryptographic assumptions for the protocol. Functions for generating fresh values, such as mkNonce, are written using the CryptoVerif primitive new that chooses a random bitstring uniformly from a type, such as the set of all nonces. Our model of Prins maintains a private database of public-private keypairs as a CryptoVerif array.

In contrast with Net and Prins, which are generic, Crypto must be written specifically for the protocol at hand; the specific definitions used for TLS are described in Section 7.

Compiling Protocol Code to CryptoVerif. The compiler first applies a series of code transformations to generate a smaller, more specialized source program. These transformations include aggressive inlining of non-recursive functions, partial evaluation of functions and patterns, and dead-code elimination.

The compiler then converts all public functions to processes, and normalizes the resulting code to fit in a restricted ML syntax that is very close to that of CryptoVerif. The final CryptoVerif script is obtained by translating these processes and the protocol security goals into CryptoVerif syntax, and by inlining abstract models for the core libraries.

ACM Journal Name, Vol. V, No. N, March 2009. 
Data structures such as records are translated to simpler forms such as tuples, and all type abbreviations are inlined. All functions that do not appear in the interfaces are eliminated, and all modules are flattened into a single module by suitably qualifying the names of functions, variables, and types. This single module then consists of datatype definitions, function definitions, and top-level code that evaluates expressions and binds variables.

Functions as Processes. For each function definition let $\mathrm{f} x=\mathrm{e}$, the translation first transforms the body expression e in continuation-passing style, into a sequence of imperative commands e': each line in e' is either a function call or a pattern match. Each line is then translated to CryptoVerif: function calls become processes that call CryptoVerif function symbols; pattern matches become let processes. Some function calls are specially translated: calls to fork spawn parallel processes; calls to log yield primitive event recording processes. Finally, the whole function definition is translated to a process of the form let $f=$ in (callf, $x) ; \ldots$; out(resultf, $r$ ) that takes its arguments on channel callf and returns its result on channel resultf. Since these channels are public, the adversary may call any of the functions in the public interface, as oracles.

Top-level Process. Each variable binding let $\mathrm{x}=\mathrm{e}$ in the source code translates to a process context that binds $\mathrm{x}$ to the result of evaluating $e$. The expression $\mathrm{e}$ is translated to a process, as expressions in function definitions, possibly spawning processes using fork. Hence, the top-level process that represents the full system consists of bindings for all variables, parallel threads for all spawned processes, and $\mathrm{N}$ replicas for each function process, where $\mathrm{N}$ is a polynomial in the security parameter.

Verification. The full CryptoVerif script consists of the computational models of the libraries, the type definitions in the protocol implementation, and the top-level process representing the oracle interface provided by the implementation to the attacker. We then write security goals as CryptoVerif queries for this process, and proceed with verification.

Besides the scripts obtained from our reference TLS implementation (Section 7), our largest case-study so far, we have extracted CryptoVerif scripts from the code of several sample protocols, including the Otway-Rees protocol and a password-based authentication protocol; we could verify both authentication properties, expressed as non-injective correspondences between events, and strong secrecy properties for keys and payloads. Although all our scripts are currently automatically verified by CryptoVerif, manual guidance may be required in general, in the form of advice.

Translation Correctness and Expressivity. To gain more confidence on the translation, we show the soundness of the translation in ongoing work [Bhargavan et al. 2009]. We define a probabilistic reduction semantics for $\mathrm{F} \#$ and use it to prove the soundness of a series of source-to-source transforms that bring our programs to a fragment of F\# closer to CryptoVerif. For this fragment, we relate our F\# semantics to a lower-level abstract machine closely related to the probabilistic polynomial semantics of CryptoVerif.

We also define abstractions for private databases and compromised keys in CryptoVerif. Private databases allow, for instance, the modelling of principal libraries (e.g., the Prins library described above) where key materials are stored. It also enables the modelling of the compromise of a principal, where its keys get leaked to the adversary. 


\section{COMPUTATIONAL VERIFICATION}

This section describes the computational security properties that we verified for our implementations of the Record and Handshake protocols. As detailed below, we verify various configurations of these protocols, but in contrast with symbolic verification, our results do not extend to the full TLS protocol.

\subsection{Record Protocol}

To verify the Record protocol (module Record in Section 4), we write some additional F\# wrapper code that sets up secure connections (module Connected), thereby abstracting the Handshake protocol.

Setting Up Connections. Module Connected has two functions for populating a database of active connections, both available to the adversary. The first function generates a fresh, private connection for the connection identifier provided by the adversary; the second function also generates a fresh connection, but it returns the whole connection content to the adversary (thus leaking its secrets) and marks the connection identifier as corrupt.

For every new connection, Connected generates fresh random secrets cr and sr, as well as a master secret ms. From these values, it then derives the encryption keys and MAC secrets. To this end, TLS relies on a pseudo random function; accordingly, we declare two cryptographic functions, prf for deriving encryption keys ek, and mprf for deriving MAC secrets mk. For example, we program the MAC key derivation as follows:

$$
\begin{array}{r}
\text { let } \mathrm{mk}=\text { mkgen }(\text { mprf }(\text { keyToBytes ms })(\text { stringToBytes “key expansion”') } \\
(\text { (concat }(\text { nonceToBytes sr })(\text { nonceToBytes cr })))
\end{array}
$$

where mkgen casts a block into a MAC secret, and mprf implements the PRF function from the following three arguments: the master secret, a TLS constant, and the concatenation of the server random and client random. (The functions stringToBytes, keyToBytes, and nonceToBytes are conversion functions; they are declared as "composable", to express that there may be inverted in polynomial time.)

Both mk and ek are then stored within the connections in the database. Module Connected provides a private lookup function to fetch these keys from a given connection identifier. This function is accessible from our code for clients and servers, but not from the adversary.

We use the tool described in Section 6 to automatically extract a computational model from the modules Record and Connected. This yields polynomially-replicated communicating sender and receiver processes, wrapped up in a context that sets up shared connections, including encryption keys and MAC secrets. To obtain the final script for verification, we compose this generated code with CryptoRecord.cv, a series of handwritten CryptoVerif declarations that model the Crypto module, including

- type definitions for the cryptographic computations;

- game equivalences that embed our security assumptions on cryptographic primitives;

- target security properties, in the form of correspondence assertions and secrecy queries.

Next, we detail these declarations.

Types for cryptography. We introduce specific types for cryptographic computations. For instance, we have types for macs (macs), mac secrets (mkey), and the seeds for generating mac keys (mkeyseed), and we declare a function fun mkgen(mkeyseed):mkey for generating a mac secret from a mac seed (derived from the connection state).

ACM Journal Name, Vol. V, No. N, March 2009. 
Security Assumptions for MAC, Symmetric Encryption, and PRF. We present our assumptions for the security primitives of the Record protocol. We refer to the libraries included in the CryptoVerif distribution for the corresponding formulations as game equivalences; see also Blanchet [2006] for other protocols that rely on these assumptions.

- MAC: The message authentication code scheme has three functions: mkgen, mac, and check for generating a mac secret from a seed, macing a message, and verifying a mac, respectively. For this scheme, we assume unforgeability under chosen message attacks (UF-CMA), stated as an equivalence that replaces all calls to mac and check, such that check performs a table lookup on any previously-generated macs instead of recomputing it. Blanchet [2006] also relies on this equivalence, and relates it to the usual formulation of UF-CMA (Proposition 2).

- Symmetric Encryption: The symmetric encryption scheme has three functions kgen, enc, and dec for generating a symmetric key from a seed, encrypting a message, and decrypting a message, respectively. Since the ciphersuites we consider use AES and DES, we model this scheme as a block cipher. We assume the notion of super pseudorandom permutation (SPRP), introduced by Phan and Pointcheval [2004], entailing that encryption is a random permutation, at least when used with randomly-chosen keys. (The "super" qualifier indicates that the adversary also has access to a decryption oracle.) SPRP is modeled as a CryptoVerif equivalence that replaces every call to encryption and decryption operations by lookups (via the CryptoVerif find command) on a table that associates previous encryption and decryption queries with freshly generated random values.

- PRF: We specify security for each of the pseudo random functions prf and mprf in the Random Oracle model [Bellare and Rogaway 1993], as an equivalence that replaces every call to the function by a table lookup, so that the first call generates a fresh random value and subsequent calls return the same value. (Blanchet and Pointcheval [2006] use a similar equivalence for proving the security of a signature scheme.)

Authentication. We consider events Send' and Recv', variants of Send and Recv used for symbolic verification in Section 5, where (for technical reasons) instead of the full connection we only record the connection identifier. We embed our authenticity property as a correspondence query in CryptoRecord.cv. The query also relies on ConnLeak events that record the identifiers of (possibly) leaked connections. Let System range over the script that consists of CryptoRecord.cv and the translation of Connected, Record, and their auxiliary modules, composed with an arbitrary polynomial adversary. CryptoVerif automatically proves record authentication on the script through 15 game transformations.

THEOREM 7 (RECORD AUTHENTICATION). With overwhelming probability, in any run of System, for any Recv' event there is a Send' event with matching record payload, or the connection is corrupted (that is, there is a ConnLeak event for its connection identifier).

Secrecy. As in Section 5.2, we extend the Record module with a function send' that, generates and sends a freshly generated record_payload over a connection. We add this function to the Record public interface, we exclude the recv function (which would otherwise act as a decryption oracle), and we disable the corruption of connections, obtaining a 
variant System ${ }^{\prime}$ of the system System of Theorem 7. CryptoVerif verifies the secrecy of record_payload, through 14 game transformations.

THEOREM 8 (RECORD SECRECY). In any run of System ${ }^{\prime}$, the sequence of values of record_payload is computationally indistinguishable from a sequence of independent random values.

In the theorem, computational indistinguishability means that a polynomial adversary can not, with overwhelming probability, distinguish the real secret from a random value. Thus, the theorem asserts that polynomial adversaries gain no information from record_payloads sent by client instances of the Record protocol.

Differences with the Symbolic Model. Symbolically, it is possible to show secrecy not only for the record payloads, but also for the encryption keys and MAC secrets used to protect the payloads. Computationally, however, we show key secrecy only before they are actually used; this is the case for the session keys of the Record protocol and for the pre-master secret of the Handshake protocol. To prove secrecy after the key is used e.g. as a session key, we would use a weaker notion such as key usability [Datta et al. 2006]. CryptoVerif does not support this notion at present, although some first models [Blanchet et al. 2008] have been tried via a combination of CryptoVerif code and then manual reasoning.

Another difference can be seen on the security notion for MACs. With CryptoVerif, the UF-CMA equivalence only states that the integrity of the MACed message is guaranteed, and says nothing on its secrecy. In contrast, symbolic models usually treat MACs as perfect one-way constructors, thereby implicitly guaranteeing message secrecy. To highlight this difference, consider a variant of the Record protocol where, instead of mac-thenencrypting, we encrypt only the payload and keep the MAC in the clear. This amounts to a one-line change in the send function, from

let $\mathrm{msg}=$ enc ek (concat payload $($ macs ToBlocks tag) $)$ in

to

let $\mathrm{msg}=$ concat (enc ek payload) (macsToBlocks tag) in

where tag is a MAC of the payload plus additional information (version and content-type). As we attempt to verify this variant, CryptoVerif fails to prove Theorem 8, since nothing guarantees that the payload remains secret. In contrast, symbolic verification still succeeds.

\subsection{Handshake Protocol}

To verify the Handshake protocol (module Handshake), we set up an environment containing a certified trusted server. More specifically, we write F\# wrapper code in a module Certified that sets up a public/private keypair of a trusted server.

Module Handshake is composed with Certified, as well as with the modules on which Handshake depends upon, most notably the Record module (albeit in null mode, as prescribed by the standard for the initial key setup). We consider two stages, which roughly correspond to the phases 2-4 of the Handshake protocol:

First stage Initially, we consider a client that sends both a ClientKeyExchange (encrypting the pre-master secret) and a Finished message (which MACs the message with a derived key from the pre-master secret). In this case, we prove secrecy of the pre-master secret, despite it being (1) encrypted for the server and (2) used as part of the key derivation in 
the subsequent Finished message (we rely both on IND-CCA2 for encryption as well as a Random Oracle assumption on the PRF, detailed below).

Second stage Subsequently, we consider a client sending the Finished message and a server processing it. In this case, we prove that the (newly established) master secret is agreed between the client and server.

As done for the Record protocol, using the tool of Section 6, we extract polynomially replicated processes from Certified, Handshake, and Record.

Security Notions for PRF and Asymmetric Encryption. We manually craft CryptoHandshake.cv with our cryptographic declarations and assumptions. We use the same cryptographic types as with the Record protocol, and include secrecy and correspondence queries. Finally, CryptoHandshake.cv includes CryptoVerif equivalences embedding our cryptographic assumptions, detailed below.

- PRF. To prove secrecy of the master secret we need the PRF to behave as a primitive that completely hides the pre-master secret from the later derived master secret. For this, as we did above in the Record protocol, we specify security of the PRF in the Random Oracle model. However, the PRF is also used as a MAC primitive in order to provide integrity of the Finished messages. In order to prove authentication of the derived master secret via the Finished messages, we also assume that the PRF primitive is UF-CMA (as explained above for the Record protocol).

- Asymmetric Encryption. We have functions skgen and pkgen for creating private and public keys; we also have functions enca and deca to encrypt and decrypt messages. (We assume a probabilistic scheme, so the encryption function inputs a seed as well.) We use a strong notion of security for asymmetric encryption, namely indistinguishability against chosen-ciphertext attacks (IND-CCA2). We use the standard equivalence of the CryptoVerif libraries, which replaces encrypted plaintexts with a message consisting of only zeroes (of the appropriate length), and replaces decryptions by table lookups.

Secrecy for the Pre Master Secret. As specified in TLS 1.0, the pre-master secret is the concatenation of a two-byte constant TLS1p0 plus 46 bytes of random. Let System ${ }^{\prime \prime}$ be the script that consists of CryptoHandshake.cv (embedding the IND-CCA2 and PRF assumptions) and the translation of Certified and Handshake (including the sending of the ClientKeyExchange and Finished messages), composed with an arbitrary polynomial adversary. CryptoVerif verifies the secrecy of random, through 18 game transformations.

THEOREM 9 (PMS RANDOM SECRECY). In any run of System ${ }^{\prime \prime}$, the sequence of random values within pre-master secrets is computationally indistinguishable from a sequence of independent random values.

Authentication of the Finished messages. By assuming UF-CMA for the PRF when used as a MAC, we can also prove the integrity the master secret, agreed between the client and server via checking of the Finished messages. We let System "' be the variant of System ${ }^{\prime \prime}$, embedding the UF-CMA assumption, and including the sending and receipt of the Finished messages. CryptoVerif verifies the necessary correspondence in 8 game transformations:

TheOREM 10 (MASTER SECRET AuthentiCATION). With overwhelming probability, in any run of System ${ }^{\prime \prime}$, for any AcceptFinished event, there is a SendFinished event with matching master secret. 


\begin{tabular}{|r|r|r|r|r|}
\hline Verification Result & F\# Code & Crypto Assumptions & Games & Time \\
\hline \hline Record Authentication (Theorem 7) & 1967 lines & 18 lines & 15 & $1.9 \mathrm{~s}$ \\
\hline Record Secrecy (Theorem 8) & 1967 lines & 25 lines & 14 & $0.3 \mathrm{~s}$ \\
\hline PMS Random Secrecy (Theorem 9) & 2497 lines & 33 lines & 18 & $1.1 \mathrm{~s}$ \\
\hline MS Authentication (Theorem 10) & 2497 lines & 23 lines & 8 & $24 \mathrm{~s}$ \\
\hline
\end{tabular}

Fig. 4. Summary of computational experimental results

Differences with the Symbolic Model. Our secrecy property is close to the symbolic notion of strong secrecy, but is finer than syntactic secrecy. For instance, symbolically, we have syntactic secrecy for the full pms with the embedded protocol version constant, not just its random part. It may also be possible to prove computational secrecy for the whole pms, as shown by Morrissey et al. [2008], if we model asymmetric encryption using a weaker one-wayness property that allows the adversary to recover some parts of pms.

Our computationally-verified models of the protocol are limited, in comparison with the symbolic ones. They do not consider resumption and server compromise, and only partly handle the composition of the Record and Handshake protocols. These limits stem from technical restrictions in the current version of our compiler and of CryptoVerif, rather than essential difficulties.

Experimental Results. Figure 4 gives the verification times for our theorems. All results are automatically proved by CryptoVerif (with no user interaction). We use FS2CV version 1.1 and CryptoVerif version 1.7. These experiments are performed on a computer with an Intel Pentium D at $3 \mathrm{GHz}$ processor.

\subsection{Related Work: Previous Computational Analyses}

There are many analyses of TLS in computational settings; we focus on the positive results, although we still mention important negative results.

Krawczyk [2001] shows that the mac-then-encrypt operation (as used in the computational analysis of our record protocol) is safe when the mac is UF-CMA and the encryption scheme is used in CBC mode and is IND-CPA. Phan and Pointcheval [2004] describe notions of PRP and SPRP (which we use for modelling in CryptoVerif) and their relation to standard semantic security and to security against active attacks.

Our treatment of (symmetric) encryption, encapsulated in the SPRP notion, assumes that exactly one block is encrypted at a time. Plaintext bitstrings are padded toward a (single, large) block that is then encrypted; the IV is assumed to be part of the key and is never updated. Hence, attacks that rely on the CBC mode, or the implementation of padding, or the choice of IVs, do not appear in our model. In practice, this means that the SPRP property may be too strong to be met by concrete implementations of the encryption primitive (e.g. AES). We leave as future work a less abstract model of symmetric encryption that accounts for the standard usages of cipher block chaining, padding, and initialization vectors.

Fouque et al. [2008] argue for the suitability of the HMACSHA1 construction as a PRF as used in TLS (whereas our model assumes a random oracle). They study randomness extraction from pre-master secret to master secret in the standard model.

Jonsson and B. S. Kaliski [2002] give a security reduction for the security of TLS/SSL when instantiated with RSA-PKCS-1v1_5 (modelling the PRF as a random oracle). This contrasts with our work in which the encryption primitive is not explicitly considered but 
assumed to be IND-CCA2. Gajek et al. [2008] present an extended random-oracle model to analyze a mutual authentication protocol built on top of TLS.

Klima et al. [2003] use the version check in the ClientKeyExchange to construct timing attacks over RSA-based sessions. In our model we do not consider side channel attacks. Padding attacks have also been exploited for the TLS protocol both for asymmetric encryption using PKCS \#1 [Bleichenbacher 1998] and for symmetric encryption in CBC mode [Yau et al. 2005]. In both cases the adversary is given an oracle that says whether plaintexts are correctly padded or not. We do not consider padding in our work.

\section{CONCLUSIONS AND FUTURE WORK}

We have presented the first symbolic and computational verification results for an interoperable TLS implementation. Using an existing model extractor and a mature verification tool, ProVerif, we proved symbolic authentication and secrecy properties for the full implementation of the Record, Handshake, and Resumption protocols. Using a novel model extractor and a recent verification tool, CryptoVerif, we also proved authentication and secrecy properties, in the more demanding computational model, for the core of the Record and Handshake protocols.

The main open area for future work is to extend computational verification to a full TLS implementation. We see three main challenges toward this goal. First, our model extractor and CryptoVerif need to be more scalable to handle the code for all three TLS protocols. Second, to compose the verification of different phases of the protocol, we would need to use a weaker notion of key secrecy, such as key usability [Datta et al. 2006] as used in recent verifications of Kerberos [Blanchet et al. 2008]. Third, our model of symmetric encryption needs to be refined to account for padding, cipher block chaining and initialization vectors.

Another area of future work is the verification of more security properties and larger applications. Our symbolic results can be extended to verify strong secrecy of keys and session secrets; although ProVerif supports such properties, they typically take more time and memory, and may require further model abstractions. TLS implementations are used within complex applications such as web browsers and web servers and the security of a session depends upon user actions such as the inspection and approval of server certificates. An open problem is to extend our symbolic and computational theorems to account for such applications, including their user interfaces.

Our results show that if a protocol implementation is carefully written, automatically verifying strong security properties against a powerful adversary is technically feasible. Verifying legacy implementations is a natural next step, but comes with its own set of practical challenges.

Acknowledgments. We thank Martín Abadi, Bruno Blanchet, Andy Gordon, and Bogdan Warinschi for their helpful comments.

\section{REFERENCES}

ABADI, M. AND Fournet, C. 2001. Mobile values, new names, and secure communication. In 28th ACM Symposium on Principles of Programming Languages (POPL'01). ACM, 104-115.

Armando, A., Basin, D. A., Boichut, Y., Chevalier, Y., Compagna, L., Cuéllar, J., Drielsma, P. H., Héam, P.-C., Kouchnarenko, O., Mantovani, J., Mödersheim, S., von Oheimb, D., Rusinowitch, M., Santiago, J., Turuani, M., Viganò, L., And Vigneron, L. 2005. The AVISPA 
Tool for the Automated Validation of Internet Security Protocols and Applications. In 17th Conference on Computer Aided Verification (CAV'05). Lecture Notes on Computer Science, vol. 3576. Springer, 281-285.

BACKeS, M. AND LAUD, P. 2006. Computationally sound secrecy proofs by mechanized flow analysis. In 13th ACM conference on Computer and Communications Security (CCS'06). ACM, 370-379.

Bellare, M., CANetTi, R., And KrawCZyK, H. 1996. Keying hash functions for message authentication. In 16th Annual Cryptology Conference on Advances in Cryptology (CRYPTO'96). Springer, 1-15.

Bellare, M. AND Rogaway, P. 1993. Random oracles are practical: A paradigm for designing efficient protocols. In 1st ACM Conference on Computer and Communications Security (CCS'93). ACM, 62-73.

Bhargavan, K., Corin, R., Fournet, C., And Zalinescu, E. 2009. Computational security for cryptographic protocol implementations. Draft.

Bhargavan, K., Fournet, C., Gordon, A. D., AND Tse, S. 2006. Verified interoperable implementations of security protocols. In 19th IEEE Computer Security Foundations Workshop (CSFW'06). IEEE Computer Society, 139-152.

BLANCHET, B. 2001. An efficient cryptographic protocol verifier based on Prolog rules. In 14th IEEE Computer Security Foundations Workshop (CSFW'01). IEEE Computer Society, 82-96.

Blanchet, B. 2002. From secrecy to authenticity in security protocols. In 9th International Static Analysis Symposium (SAS'02). Lecture Notes in Computer Science, vol. 2477. Springer, 342-359.

BLANCHET, B. 2006. A computationally sound mechanized prover for security protocols. In IEEE Symposium on Security and Privacy. IEEE Computer Society, 140-154.

BLANCHET, B. 2007. Computationally sound mechanized proofs of correspondence assertions. In 20th IEEE Computer Security Foundations Symposium (CSF'07). IEEE Computer Society, 97-111.

Blanchet, B., JagGard, A. D., Scedrov, A., And Tsay, J.-K. 2008. Computationally sound mechanized proofs for basic and public-key Kerberos. In ACM Symposium on Information, Computer and Communications Security (ASIACCS'08). ACM, 87-99.

Blanchet, B. And Pointcheval, D. 2006. Automated security proofs with sequences of games. In 26th Annual International Cryptology Conference (CRYPTO'06). Lecture Notes on Computer Science, vol. 4117. Springer, 537-554.

BLEICHENBACHER, D. 1998. Chosen ciphertext attacks against protocols based on RSA encryption standard PKCS \#1. In 18th Annual Cryptology Conference on Advances in Cryptology (CRYPTO'98). Lecture Notes in Computer Science, vol. 1462. Springer, 1-12.

CHAKI, S. AND DATTA, A. 2008. ASPIER: An automated framework for verifying security protocol implementations. Technical Report CMU-Cylab-08-012, Carnegie Mellon University.

CISCO. 2007. SSL/TLS certificate and SSH public key validation vulnerability. http://www.cisco.com/warp/public/707/cisco-sa-20070118-certs.shtml.

CORIN, R. AND DEN HARTOG, J. 2006. A probabilistic hoare-style logic for game-based cryptographic proofs. In 33rd International Colloquium on Automata, Languages and Programming, Part II (ICALP'06). Lecture Notes in Computer Science, vol. 4052. Springer, 252-263.

Datta, A., Derek, A., Mitchell, J. C., And Warinschi, B. 2006. Computationally sound compositional logic for key exchange protocols. In 19th IEEE workshop on Computer Security Foundations (CSFW'06). IEEE Computer Society, 321-334.

Díaz, G., Curtero, F., Valero, V., And Pelayo, F. 2004. Automatic verification of the TLS handshake protocol. In 19th ACM Symposium on Applied Computing (SAC'04). ACM, 789-794.

DierKs, T. AND ALLEN, C. 1999. The TLS protocol version 1.0.

Dier Ks, T. And Rescorla, E. 2006. The Transport Layer Security (TLS) Protocol Version 1.1.

Dierks, T. AND Rescorla, E. 2008. The Transport Layer Security (TLS) Protocol Version 1.2.

DiETRICH, S. 1997. A formal analysis of the secure sockets layer protocol. Ph.D. thesis, Adelphi University.

Dolev, D. AND YAO, A. 1983. On the security of public key protocols. IEEE Transactions on Information Theory IT-29, 2, 198-208.

Fouque, P.-A., Pointcheval, D., And Zimmer, S. 2008. HMAC is a randomness extractor and applications to TLS. In ACM Symposium on Information, Computer and Communications Security (ASIACCS'08). ACM, 21-32.

Frier, A. O., Karlton, P., AND Kocher, P. C. 1996. The SSL protocol version 3.0. Internet Draft, IETF. 
Gajek, S., Manulis, M., SAdeghi, A.-R., And Schwenk, J. 2008. Provably secure browser-based user-aware mutual authentication over TLS. In ACM Symposium on Information, Computer and Communications Security (ASIACCS'08). ACM, 300-311.

Goubault-LarrecQ, J. AND Parrennes, F. 2005. Cryptographic protocol analysis on real C code. In 6 th International Conference on Verification, Model Checking and Abstract Interpretation (VMCAI'05). Lecture Notes in Computer Science, vol. 3385. Springer, 363-379.

He, C., Sundararajan, M., Datta, A., Derek, A., and Mitchell, J. C. 2005. A modular correctness proof of IEEE $802.11 \mathrm{i}$ and TLS. In 12th ACM conference on Computer and Communications Security (CCS'05). ACM, 2-15.

HICKMAN, K. E. 1995. The SSL protocol. Draft specification, Netscape.

JONSSON, J. AND B. S. KALISKI, J. 2002. On the security of RSA encryption in TLS. In 22nd Annual International Cryptology Conference (CRYPTO'02). Lecture Notes on Computer Science, vol. 2442. Springer, 127-142.

JÜRJENS, J. 2006. Security analysis of crypto-based java programs using automated theorem provers. In 21st IEEE/ACM International Conference on Automated Software Engineering (ASE'06). IEEE Computer Society, 167-176.

KamiL, A. AND Lowe, G. 2008. Analysing TLS in the strand spaces model. Tech. rep., Oxford University Computing Laboratory.

Klima, V., Pokorny, O., And Rosa, T. 2003. Attacking RSA-based sessions in SSL/TLS. In Cryptographic Hardware and Embedded Systems (CHES'03). Lecture Notes on Computer Science, vol. 2779. Springer, 426-440.

KRAWCZYK, H. 2001. The order of encryption and authentication for protecting communications (or: How secure is SSL?). In 21st Annual International Cryptology Conference on Advances in Cryptology (CRYPTO'01). Lecture Notes on Computer Science, vol. 2139. Springer, 310-331.

LAUD, P. 2005. Secrecy types for a simulatable cryptographic library. In 12th ACM conference on Computer and Communications Security (CCS'05). ACM, 26-35.

Microsoft 2002. Microsoft .NET Framework. Microsoft. http://www.microsoft.com/NET/.

Mitchell, J. C., Shmatikov, V., AND Stern, U. 1998. Finite-state analysis of SSL 3.0. In 7th USENIX Security Symposium (SSYM'98). USENIX Association, 201-216.

MORRISSEY, P., SMART, N., AND WARINSCHI, B. 2008. A modular security analysis of the TLS handshake protocol. In 14th Annual International Conference on the Theory and Application of Cryptology and Information Security (ASIACRYPT'08). Lecture Notes on Computer Science, vol. 5350. Springer, 55-73.

NeEdHAM, R. AND SCHROEDER, M. 1978. Using encryption for authentication in large networks of computers. Communications of the ACM 21, 12, 993-999.

Ogata, K. AND Futatsugi, K. 2005. Equational approach to formal analysis of TLS. In 25th IEEE International Conference on Distributed Computing Systems (ICSCS'05). IEEE Computer Society, 795-804.

OPENSSL. 2009. evp_verifyfinal function signature verification vulnerability. http://web.nvd.nist.gov/view/vuln/detail?vulnId=CVE-2008-5077.

PAULSON, L. C. 1999. Inductive analysis of the Internet protocol TLS. ACM Transactions on Information and System Security 2, 3, 332-351.

Phan, D. H. And Pointcheval, D. 2004. About the security of ciphers (semantic security and pseudo-random permutations). In 11th International Workshop on Selected Areas in Cryptography (SAC'04). Lecture Notes in Computer Science, vol. 3357. Springer, 182-197.

RUBY. 2006. Net:https certificate validation vulnerability. http: //web.nvd.nist.gov/view/vuln/detail?vulnId=CVE-2007-5162.

SyME, D. 2005. F\#. Microsoft Research. http://research.microsoft.com/fsharp/.

TSAHHIROV, I. AND LAUD, P. 2007. Application of dependency graphs to security protocol analysis. In $3 r d$ Symposium on Trustworthy Global Computing. Lecture Notes in Computer Science, vol. 4912. Springer, 294-311.

WAGNER, D. AND SCHNEIER, B. 1996. Analysis of the SSL 3.0 protocol. In 2nd USENIX Workshop on Electronic Commerce (WOEC'96). USENIX Association, 29-40.

Yau, A. K. L., Paterson, K. G., And Mitchell, C. J. 2005. Padding oracle attacks on CBC-mode encryption with secret and random IVs. In Fast Software Encryption. Springer, 299-319. 